\title{
Hémangioendothéliome épithélioïde
}

\section{Epithelioid Hemangioendothelioma}

\section{SOphie COUSIN ${ }^{1}$, François LE LOARER ${ }^{2}$, Amandine CROMBÉ ${ }^{3}$, Marie KARANIAN ${ }^{4}$, Véronique MINARD-COLIN ${ }^{5}$, Nicolas PENEL ${ }^{6,7}{ }^{*}$}

1 - Département d'Oncologie Médicale, Institut Bergonié, 229 Cours de l'Argonne, 33000 Bordeaux

2 - Département d'Anatomopathologie, Institut Bergonié, 229 Cours de l'Argonne, 33000 Bordeaux

3 - Département d'Imagerie Médicale, Institut Bergonié, 229 Cours de l'Argonne, 33000 Bordeaux

4 - Département d'Anatomopathologie, Centre Léon Bérard, 28 Promenade Léa et Napoléon Bullukian, 69008 Lyon

5 - Département d'Oncologie Pédiatrique, Institut Gustave Roussy, 114 Rue Edouard Vaillant, 94800 Villejuif

6 - Département d'Oncologie Médicale, Centre Oscar Lambret, 3 Rue Frédéric Combemale, 59000 Lille

7 - Service d’Oncologie Médicale, Hôpital Claude Huriez, Rue Michel Polonowski, 59000 Lille

* Pour correspondance : Nicolas PENEL

Département de Cancérologie Générale, Centre Oscar Lambret, 59020 Lille, France Mail.n-penel@o-lambret.fr 


\title{
Hémangioendothéliome épithélioïde
}

\author{
Epithelioid Hemangioendothelioma
}

\begin{abstract}
Les auteurs décrivent ici les présentations cliniques, les arguments diagnostics radiologiques et anatomopathologiques, le profil évolutif et le traitement des hémangioendothéliomes épithélioïdes. La grande majorité des hémangioendothéliomes épithélioïdes (90\%) présentent une translocation chromosomique réciproque $\mathrm{t}(1 ; 3)(\mathrm{p} 36 ; \mathrm{q} 23-25)$ associée à la synthèse d'une protéine de fusion WWTR1/CAMTA1. Ces tumeurs vasculaires de bas grade peuvent être localisées ou d'emblée multifocales. Elles sont souvent lentement évolutives. Localisées, elles appellent un traitement local. Multifocales, elles nécessitent tout d'abord une surveillance attentive. Certaines d'entre elles peuvent être spontanément stables pendant des années voire des décennies. En cas de progression, il n'y a pas de standard thérapeutique. Le diagnostic doit être confirmé au sein d'un réseau spécialisé de bio pathologie (RRePS) et la prise en charge interdisciplinaire relève des centres sarcomes labélisés par I'Institut National du Cancer (NetSarc).
\end{abstract}

Mots-clés : centre de référence, diagnostic, hémangioendothéliomes épithélioïdes, sarcome, traitement

\section{Abstract}

We describe herein the inaugural manifestations, the radiological and histological diagnosis criteria for and the outcome of epithelioid hemangioendothelioma (EHE). Most of EHE (90\%) display a specific reciprocal chromosomic translocation $\mathrm{t}(1 ; 3)(\mathrm{p} 36 ; \mathrm{q} 23-25)$, which is associated with the synthesis of fusion protein WWTR1/CAMTA1. EHE are low grade vascular sarcomas. EHE could be initially localized or multifocal. At localized stage, EHE are best treated with focal treatments. At multifocal stage, the upfront strategy is watchful follow-up. Some multifocal EHE display very indolent course with spontaneous stable disease for years or decades. In case of progressive multifocal EHE, there is no consensual treatment. Diagnostic and clinical management of EHE requires interdisciplinary expertise from labeled centers.

Keywords : diagnosis, epithelioid hemangioendothelioma, management, reference centers, sarcoma

\section{DIAGNOSTIC ANATOMO-PATHOLOGIQUE ET MOLÉCULAIRE}

L'hémangioendothéliome épithélioïde (HE) est une entité de description récente. Il s'agit d'une tumeur vasculaire rare. Les critères diagnostiques initiaux ont été fixés dans les années 1970-1980. Il s'agit de tumeurs ubiquitaires : les hémangioendothéliomes épithélioïdes peuvent se développer au niveau des parties molles, des viscères (foie et poumon) ou les os. En 1975, Dail et Liebow ont décrit le premier cas d'hémangioendothéliome épithélioïde pulmonaire comme une forme agressive de carcinome bronchiolo-alvéolaire, infiltrant massivement les vaisseaux sanguins et les petites voies respiratoires. Ils avaient alors nommé cette entité "tumeur intravasculaire bronchiolo-alvéolaire" [1]. Cet aspect $d^{\prime}$ invasion vasculaire et d'infiltration initialement décrit demeure un critère diagnostique important de 
I'hémangioendothéliome épithélioïde. Plus tard, Weiss et al. ont introduit le terme d' « hémangioendothéliome épithélioïde » [Epithelioid hemangioendothelioma] pour décrire une tumeur des parties molles ou osseuses, pouvant avoir des aspects bénins (« hémangiome ») ou malins («angiosarcomes») [2]. Dans les années 1970, Corrin et al. ont démontré que les hémangioendothéliomes épithélioïdes dérivaient des progéniteurs endothéliaux [3]. Weldon-Linne et al. ont établi la présence d'une expression d'antigènes liés au facteur VII [4]. Les hémangioendothéliomes épithélioïdes expriment d'autres marqueurs endothéliaux. Sur le plan moléculaire, la présence d'une translocation chromosomique réciproque est un critère diagnostique majeur $(t(1 ; 3)(p 36.3 ; q 25))$, translocation absente en cas d'hémangiome [5]. Dans la classification actuelle de l'Organisation Mondiale de la Santé, les hémangioendothéliomes épithélioïdes sont classés parmi les tumeurs localement agressives avec potentiel métastatique [6].

La translocation $\mathrm{t}(1 ; 3)(\mathrm{p} 36 ; \mathrm{q} 23-25)$ est l'événement carcinologique initial, dans la majorité des cas. Cette translocation amène à la synthèse d'une protéine hybride WWTR1-CAMTA1 [5, 7]. WWTR1 code un co-activateur transcriptionnel, exprimé de manière intense au niveau des cellules endothéliales, et qui stimule la différenciation des cellules souches mésenchymateuses [8]. CAMTA1 code un facteur de transcription fixant le calmoduline $[5,8]$. Errani et al. ont montré que les hémangioendothéliomes épithélioïdes sont des proliférations clonales, qui présentent tous les mêmes points de translocation [7]. La protéine hybride WWTR1-CAMTA1 amène à une translocation nucléaire de CAMTA1, et une activation constitutive de la voie Hippo [9].

Environ $10 \%$ des hémangioendothéliomes épithélioïdes présentent une autre translocation $(t(11 ; X)(q 13 ; p 11.22))$ impliquant YAP1 et TFE3. YAP1 code un co-ativateur transcriptionnel. TFE3 code un facteur de transcription (microphthalmia transcription factor). Ces hémangioendothéliomes épithélioïdes sont particuliers : sujets plus jeunes et aspect vasculaire plus mature avec des vaisseaux bien formés et solides [10-12].

Les critères diagnostiques sont résumés dans le tableau 1.

\section{ÉPIDÉMIOLOGIE}

Au sein de la ContiCabase (base de données multicentrique des sarcomes du Groupe Sarcome Français), parmi les 10262 cas de sarcomes, on note 42 cas d'hémangioendothéliome épithélioïde, soit $0,4 \%$ de l'ensemble des sarcomes. Il s'agit donc d'une pathologie tout à fait exceptionnelle. Les hémangioendothéliomes épithélioïdes peuvent être diagnostiqués à tout âge, mais l'âge médian au diagnostic est habituellement de 20 à 30 ans. Il y a autant de cas chez les hommes que chez les femmes.

\section{TABLEAUX RADIO-CLINIQUES}


Les hémangioendothéliomes épithélioïdes sont hétérogènes dans leur présentation.

\section{hémangioendothéliomes épithélioïdes hépatiques (Figure 1)}

Environ $20 \%$ des hémangioendothéliomes épithélioïdes se développent au niveau hépatique. Deux tiers des hémangioendothéliomes épithélioïdes hépatiques surviennent chez des femmes, avec un âge médian de 45 ans [17]. Un quart des hémangioendothéliomes épithéliö̈des hépatiques sont asymptomatiques. Les symptômes révélateurs sont non spécifiques comme la perte de poids, la fièvre, la fatigue et l'ictère [18-20]. Cependant le symptôme le plus fréquent est l'hépatalgie [17, 19]. Certains tableaux dramatiques peuvent être révélateurs comme un syndrome de Budd-Chiari (thrombose des veines sus-hépatiques), syndrome de Kasabach-Meritt (thrombopénie par trappage vasculaire) ou syndrome hémorragique [21-22]. Environ $50 \%$ des hémangioendothéliomes épithélioïdes hépatiques sont d'emblée métastatiques, avec atteinte pulmonaire ou osseuse [17]. Les données de la littérature concernant l'imagerie des hémangioendothéliomes épithélioïdes hépatiques sont limitées. Il s'agit habituellement de lésions unies ou multifocales lobulées périphériques [20]. Les tumeurs sont calcifiées dans 13 à $20 \%$ des cas. On observe un aspect de rétraction de la capsule hépatique sans 11 à $25 \%$ des cas, pour les lésions périphériques. Ces masses ont tendance à confluer avec un aspect de compensation hypertrophique du foie sain [20,23, 24] A l'échographie les hémangioendothéliomes épithélioïdes sont hypoéchogènes. [25-26]. Au scanner sans injection, les hémangioendothéliomes épithélioïdes présentent une faible densité. Après injection de produit de contraste, la prise de contraste est progressive, plutôt périphérique, avec aspect retardé d'une prise de contraste homogène. Cet aspect peut être confondu avec un hémangiome et son classique rehaussement «en motte» [25]. Cependant d'autres aspects sont possibles avec des prises de contraste partielles, focales, sur de fines portions, parfois en étroit anneau. Cette prise de contraste est inconstante [26-27]. À I'IRM, les hémangioendothéliomes épithélioïdes hépatiques sont en hyposignal $\mathrm{T} 1$ et modérément en hypersignal $\mathrm{T} 2$ hétérogène [26]. Le rehaussement après injection intraveineuse de chélates de Gadolinium est là encore progressif, centripète [27].

Aucun de ces aspects n'est spécifique et le diagnostic différentiel est difficile avec les hémangiomes atypiques, les métastases hépatiques et les cholangiocarcinomes hépatiques.

\section{Les hémangioendothéliomes épithélioïdes pulmonaires (Figure 2)}

Environ $10 \%$ des hémangioendothéliomes épithélioïdes se développent au niveau pulmonaire. Deux tiers de ces hémangioendothéliomes épithélioïdes pulmonaires surviennent chez les femmes, avec un âge médian au diagnostic de 40 ans. Environ $50 \%$ des hémangioendothéliomes épithélioïdes pulmonaires sont découverts de manière fortuite sur une imagerie thoracique. Les symptômes inauguraux sont aspécifiques, comme la fièvre, la perte de poids, des pleurodynies, des hémoptysies et l'hémorragie alvéolaire [28]. 
La description des aspects radiologiques des hémangioendothéliomes épithélioïdes pulmonaires repose sur quelques courtes séries rétrospectives. Le scanner est l'examen clé. Trois aspects ont été décrits : 1) l'aspect multinodulaire (fait de petites lésions périvasculaires de moins de $2 \mathrm{~cm}$ périvasculaires, parfois lobulées, voire calcifiées), 2) l'aspect réticulonodulaire multifocal et 3) l'atteinte pleurale avec rehaussement modéré après injection [29, 30]. Les hémoptysies, les épanchements pleuraux et I'aspect non nodulaire sont de mauvais pronostics [28, 31, 32].

\section{Les hémangioendothéliomes épithélioïdes multifocaux}

$20 \%$ des hémangioendothéliomes épithéliö̈des sont multifocaux au diagnostic, avec atteintes hépatique et pulmonaire. Ces formes multifocales peuvent être asymptomatiques. Les symptômes inauguraux peuvent être l'altération de l'état général, la douleur, la fièvre, l'anémie hémolytique et les coagulopathies de consommation [33].

\section{Les hémangioendothéliomes épithélioïdes des parties molles (Figure 3)}

De siège ubiquitaire, ces masses lentement évolutives, peu symptomatiques se développent au niveau des extrémités. Ces hémangioendothéliomes épithélioïdes des parties molles peuvent être de siège sus- ou sous-aponévrotiques. Ces tumeurs sont développées à proximité des vaisseaux dans 50 à $70 \%$ des cas, avec parfois obstruction de la lumière vasculaire. À I'IRM il s'agit de masses hétérogènes, notamment après injection de produit de contraste. On observe parfois des calcifications, des hémorragies spontanées, des œdèmes périphériques et des érosions osseuses [34].

\section{Les hémangioendothéliomes épithélioïdes osseux (Figure 4)}

Il s'agit de lésions ostéolytiques développées au niveau de la corticale, avec parfois rupture de la corticale et extension dans les parties molles. Les principaux symptômes sont la douleur, la tuméfaction et les compressions, notamment neurologiques en cas de localisation vertébrale. Dans $14 \%$ des cas les hémangioendothéliomes épithélioïdes sont isolés au niveau osseux, ou au contraire s'intégrer à une forme multifocale. La plupart des hémangioendothéliomes épithéliö̈des osseux affectent les os longs des membres et plus rarement les vertèbres (moins de $10 \%$ des cas). Des atteintes multiples peuvent être observées sur une seule pièce osseuse, ou atteindre plusieurs segments osseux soit dans une région anatomique, soit de manière diffuse sur l'ensemble du squelette. L'aspect au scanner ou à l'IRM n'est pas spécifique avec des lésions ostéolytiques bien limitées sans réaction périostée. Les calcifications sont possibles. La prise de contraste est hétérogène. Les fractures pathologiques sont possibles [35, 36].

\section{Les formes pédiatriques}


Les cas pédiatriques sont exceptionnels [19]. Vingt-quatre cas d'hémangioendothéliome épithélioïde hépatiques ou pulmonaires ont été diagnostiqués chez des patients de moins de 18 ans. L'âge médian est de 12 ans (4 à 18 ans). Pour le seul cas analysé sur le plan moléculaire, la présence du transcrit de fusion est documenté. La présence d'un épanchement pleural est de mauvais pronostic, avec la survenue du décès dans l'année pour les 2 patients présentant ces épanchements. Les analyses moléculaires n'ont pas identifié jusqu'à d'altération génomique "actionnables". Si cela est possible la résection de la maladie (y compris par transplantation hépatique) est à discuter [37].

\section{ÉVOLUTION}

Les hémangioendothéliomes épithélioïdes sont considérés comme des tumeurs de malignité intermédiaire dans la classification OMS 2013 [6]. Leur évolution n'est pas prévisible, entre comportement « bénin » et «malin ». Après exérèse d'une tumeur isolée, le risque de métastase est augmenté en cas de taille supérieure à $3 \mathrm{~cm}$, au moins 3 mitoses par 50 champs. La survie sans maladie est de $100 \%$ pour les tumeurs ne présentant pas ces critères et de $59 \%$ pour les tumeurs présentant l'un de ces 2 critères [38].

Certaines formes multifocales sont stables pendant des années voire des décennies. Après la mise en évidence d'une progression, la survie globale est de 1,3 an. Les facteurs pronostics péjoratifs sont l'altération de l'état général, I'anémie (notamment hémolytique), la coagulopathie de consommation et l'apparition d'ascite ou d'épanchement pleural [32, 39, 40].

\section{PRISE EN CHARGE}

Il s'agit d'une pathologie rare, appelant une confirmation diagnostic et une prise en charge interdisciplinaire au sein de centres experts labelisés (RRePS pour le diagnostic anatomo-pathologique et moléculaire et NetSarc pour la prise en charge clinique).

\section{Diagnostic}

Le diagnostic anatomopathologique est difficile. Une $2^{\mathrm{e}}$ lecture par un expert et la recherche de la translocation $\mathrm{t}(1 ; 3)(\mathrm{p} 36 ; \mathrm{q} 23-25)$ doivent être recommandées.

\section{Bilan d'extension}

On peut recommander une IRM avec séquences pondérées $T 1, T 2$, sans et avec suppression de graisse puis T1 après injection intraveineuse de chélates de Gadolinium incluant au moins 2 plans 
orthogonaux pour les lésions des parties molles, et un scanner thoraco-abdomino-pelvien avec injection intraveineuse de produit de contraste iodé. La place de scintigraphie osseuse n'est pas clairement établie. Ce bilan complet est recommandé avant de discuter de toute option chirurgicale. Le rôle de la tomographie par émission de positions au 18 FDG n'est pas clairement établi. La fixation est inconstante, l'intensité est variable (Figure 5) [41-45].

\section{$\underline{\text { Chirurgie }}$}

Quand cela est possible, l'exérèse atypique est recommandée pour les lésions pulmonaires isolées. On ne recommande pas de curage lymphatique puisque les patients présentant un envahissement ganglionnaire sont exceptionnels [28, 31]. Il n'y a pas de données concernant la décortication ou la résection pleurale pour les atteintes pleurales.

Les atteintes hépatiques isolées peuvent être traitées par chirurgie. Mehrabi et al. ont rapporté le devenir de 8 patients traités soit par hépatectomie ( 3 patients) soit par transplantation hépatique ( 5 cas). Après un suivi médian de 100 mois, tous les patients étaient en vie, mais 3 présentaient une récidive dont 2 au niveau hépatique. Les récidives ont été observées pour 1 des 3 patients traités par hépatectomie et 2 des 5 patients traités par transplantation hépatique [19]. Thomas et al. ont rapportés le devenir de 7 patients traités par hépatectomie. Après un suivi médian de $5 \mathrm{~A}$ mois, 3 n'ont pas présenté de récidive, 3 ont présenté une récidive (avec un décès), et un patient n'a pas présenté de rechute mais est décédé d'une autre cause.

Dans une étude rétrospective de 50 cas [17], Thomas et al. n'ont pas mis en évidence de différence de survie globale entre les patients traités par surveillance attentive (25 patients), ceux traités par chirurgie ( 7 cas), embolisation ou traitement systémique (18 patients). Les données de la littérature sont résumées dans le tableau 2.

Les hémangioendothéliomes épithélioïdes osseux peuvent nécessiter une résection large avec reconstruction articulaire, geste préventif de stabilisation ou radiofréquence [35, 50].

\section{Radiothérapie}

Il a été observé la survenue de sarcomes en territoire irradié après traitement de $8 \%$ des hémangioendothéliomes épithélioïdes osseux. La radiothérapie est donc réservée aux tumeurs localisées non opérables [35]. Il n'y a pas de donnée concernant le traitement des hémangioendothéliomes épithélioïdes pulmonaires par radiothérapie [28]. La radiothérapie est rarement utilisée pour les hémangioendothéliomes épithélioïdes hépatiques [51, 19], la radiothérapie symptomatique peut être utilisée. 


\section{La surveillance attentive}

Parce que les hémangioendothéliomes épithélioïdes peuvent être spontanément stable, la surveillance attentive est survenant l'approche à privilégier pour les maladies inopérables et multifocales. Des cas de régression spontanée ont été documentés [52-54]. Yousaf et al. ont rapport le devenir de 4 patients avec hémangioendothéliomes épithélioïdes multifocaux surveillés ; après un suivi de 60 mois, 1 seul patient de 85 ans est décédé de l'évolution tumorale après 10 ans [55]. Thomas et al. ont décrit le devenir de 25 patients avec hémangioendothéliomes épithélioïdes hépatiques surveillés. Une progression a été observée dans 14 cas, après un suivi médian de 322 jours. Un de ces patients avec progression est décédé rapidement de l'évolution tumorale. Les 13 autres patients ont été traités avec différentes approches : chirurgie ( 2 cas), traitements systémiques ( 8 cas) et traitements focaux ( 3 cas), dont radiofréquence, embolisation et injection intra-tumorale. Ces auteurs recommandent une surveillance attentive avant d'envisager un geste d'hépatectomie [17].

\section{$\underline{\text { Traitements systémiques }}$}

Lorsque les patients présentent des signes cliniques (altération de l'état général, fièvre, anémie hémolytique, troubles de la coagulation, épanchements pleuraux ou péritonéaux), il convient d'envisager un traitement systémique, mais il n'y a pas de consensus à ce sujet.

Il n'y a pas d'essai clinique ayant évalué un protocole de chimiothérapie dans cette indication. La doxorubicine peut être recommandée comme pour tout sarcome. Cependant, Youssaf et al. n'ont rapporté aucune réponse objective pour 6 patients traités par doxorubicine et 2 patients traités par doxorubicine liposomiale [55]. À I'opposé, deux autres auteurs ont rapporté chacun une réponse objective sous doxorubicine liposomiale. Kelly et $\mathrm{O}^{\prime} \mathrm{Neil}$ ont décrit le cas d'un patient atteint d'hémangioendothéliome épithélioïde multifocal avec atteinte osseuse, traité par doxiorubicine liposomiale $45 \mathrm{mg} / \mathrm{m}^{2}$ toutes les 3 semaines pendant 20 mois. La maladie a été contrôlée pendant 24 mois avant que ne survienne une rapide dégradation de l'état général [56]. Grander et al. ont rapporté une réponse partielle ayant duré 18 mois chez un patient atteint d'hémangioendothéliome épithélioïde hépatique [57].

Yousaf et al. ont rapporté l'activité du paclitaxel hebdomadaire chez 8 patients. La durée médiane de traitement est de 3 mois, sans réponse objective; mais avec des stabilisations prolongées avec amélioration symptomatique chez 4 patients [55].

En ce qui concerne les autres protocoles, il a été rapporté une stabilisation prolongée de 72 mois avec la gemcitabine chez un patient présentant une maladie réfractaire à l'association doxorubicine/ifosfamide [58], une réponse partielle (de $90 \%$ ) avec l'association carboplatin/pemetrexed/bevacizumab [59], et une stabilité pour 1 des 3 patients traités par cyclophosphamide/étoposide ou cyclophosphamide/vinblastine [55]. 
Il existe une certaine logique à évaluer le rôle thérapeutique des anti-angiogéniques, ceci dit les données précliniques sont assez limitées [60].

Le thalidomide a été évalué chez 8 patients, avec 2 réponses partielles, 1 stabilité et 5 progressions [55, 61-64]. L'activité du pazopanib a été rapportée par l'EORTC chez 10 patients avec une réponse complète, 1 réponse partielle, 4 stabilités et 3 progressions (et un patient non évaluable). La survie sans progression était de 26 mois [63]. Un autre cas de stabilité prolongée ( 8 ans) a été rapporté avec le pazopanib [65].

Le Groupe Sarcome Français a conduit une étude de phase II évaluant le sorafenib chez 15 patients atteints d'hémangioendothéliome épithélioïde. La durée médiane du traitement était de 124 jours. Les taux de non-progression à 2, 4 et 6 mois étaient de $84 \%(11 / 13), 46 \%(6 / 13)$ et $38 \%(5 / 13)$. Il a été observé 2 réponses partielles ayant duré 2 et 9 mois [66]. D’autres cas ont été rapportés en dehors de cet essai [67-68].

Stacchiotti et al. ont rapporté l'activité du sirolimus chez 17 patients atteints d'hémangioendothéliome épithélioïde. Un patient a présenté une réponse partielle, 12 une stabilité et 3 une progression tumorale. Le temps median jusqu'à progression est de 12 mois (1-45 mois). La survie médiane est de 16 mois [68].

Enfin, 7 patients atteints d'hémangioendothéliome épithélioïde ont été inclus dans un essai de phase II testant l'activité du bevacizumab. Deux patients ont présenté une réponse partielle et un patient une progression rapide. Le nombre médian de cycle administré est de 17 (soit 52 semaines). Le temps médian jusqu'à progression est de 39 semaines et la survie médiane de 142 semaines. [69].

\section{Stratégie thérapeutique}

Il s'agit d'une pathologie exceptionnelle. On peut recommander une confirmation du diagnostic par un expert pathologiste. En cas de lésion isolée, accessible à un geste chirurgical à visée curative, la chirurgie peut être proposée. En cas de lésion inopérable ou multifocale, la surveillance attentive peut être proposée en première intention.

L'objectif d'un traitement systémique est palliatif [70], visant à freiner l'évolution de la maladie et maintenir la qualité de vie. Il n'y a pas de traitement systémique standard. Certains signes (altération de l'état général, fièvre, anémie notamment hémolytique, coagulopathie de consommation, hémoptysies, épanchements pleuraux et péritonéaux) témoignent d'une aggravation tumorale et appellent un traitement symptomatique.

\section{PERSPECTIVES}


Des études multicentriques internationales sont indispensables pour mieux connaître cette maladie exceptionnelle. Les essais cliniques sont trop rares. De manière récente ont été mis en évidence des altérations de FGF-R et ROS1 qui pourraient permettre d'envisager des traitements ciblés [71].

\section{Les auteurs n'ont pas de conflit d'intérêts à déclarer.}

\section{Références}

1 - Dail DH, Liebow AA. Intravascular bronchioloalveolar tumor. Am J Pathol 1975;78:6a-7a

2 - Weiss SW, Ishak KG, Enzinger FM, Sweet DE, Enzinger FM. Epithelioid hemangioendothelioma and related lesions. Semin Diagn Pathol 1986;3:259-87.

3- Corrin B, Manners B, Millard M, Weaver L. Histogenesis of the so-called 'intravascular bronchioloalveolar tumor'. J Pathol 1979;123:163-7.

4 - Weldon-Linne CM, Victor TA, Christ ML, Fry WA. Angiogenic nature of the intravascular bronchioloalveolar tumor of the lung: an electron microscopic study. Arch Pathol 1981;105:174-9.

5 - Errani C, Zhang L, Antonescu CR, Hajdu M, Singer S, Maki RG, et al. À novel WWTR1-CAMTA1 gene fusion is a consistent abnormality in epithelioid hemangioendothelioma of different anatomic sites. Genes Chromosomes. Cancer 2011;50:644-53.

6 - WHO Classification of Tumours, Volume 5 IARC WHO Classification of Tumours, N5 Fletcher CDM, Bridge JA, Hogendoorn P, Mertens F, IARC

7 - Errani C, Sung YS, Zhang L, Healey JH, Antonescu CR. Monoclonality of multifocal epithelioid hemangioendothelioma of the liver by analysis of WWRT1-CAMTA1 breakpoints. Cancer Genetic 2012; 470:12-17

8 - Tanas MR, Sboner A, Oliveira AM, Erickson-Johnson MR, Hespelt J, Hanwright PJ, et al.: Identification of disease-defining gene fusion in epithelioid hemangioendothelioma. Sci Transl Med 2011, 3:98ra82.

9 - Tanas MR, Ma S, Jadaan FO, Ng CK, Weigelt B, Reis-Filho JS et al. Mechanism of action of a WWTR1(TAZ)-CAMTA1 fusion oncoprotein. Oncogene. 2016; Feb 18;35(7);929-38

10 - Antonescu CR, Le Loarer F, Mosquera JM, Sboner A, Zhang L, Chen CL, et al. Epithelioid hemangioendotheliomas with TFE3 gene translocaion are compossible wih CAMTA1 rearrangements. OncoTargets 2016;7:7480-88

11- Flucke U, Vogels RJ, de Saint Aubain Somerhausen N, Creytens DH, Riedl RG, van Gorp JM, et al. Epithelioid Hemangioendothelioma: clinicopathologic, immunohistochemical, and molecular genetic analysis of 39 cases. Diagnostic pathology. 2014; 9:131.

12 - Patel NR, Salim AA, Sayeed H, Sarabia SF, Hollingsworth F, Warren M et al. Molecular characterization of epithelioid Haemangioendotheliomas identifies novel WWTR1-CAMTA1 fusion variants. Histopathology 2015:67:699-708 
13 - Mentzel T, Beham A, Calonje E, Katenkamp D, Fletcher CD. Epithelioid hemangioendothelioma of skin and soft tissues: clinicopathologic and immunohistochemical study of 30 cases. Am J Surg Pathol. avr 1997;21(4):363-74.

14 - Shibuya R, Matsuyama A, Shiba E, Harada H, Yabuki K, Hisaoka M. CAMTA1 is a useful immunohistochemical marker for diagnosing epithelioid haemangioendothelioma. Histopathology. déc 2015;67(6):827-35.

15 - Doyle LA, Fletcher CDM, Hornick JL. Nuclear Expression of CAMTA1 Distinguishes Epithelioid Hemangioendothelioma From Histologic Mimics. Am J Surg Pathol. janv 2016;40(1):94-102.

16 - Fukayama M, Nihei Z, Takizawa T, Kawaguchi K, Harada H, Koike M. Malignant epithelioid hemangioendothelioma of the liver, spreading through the hepatic veins. Virchows Arch A Pathol Anat Histopathol. 1984;404(3):275-87.

17 - Thomas RM, Aloia TA, Truty MJ, Tseng WH, Choi EA, Curley SA et al. Treatment sequencing strategy for hepatic epithelioid hemangioendothelioma. HPB 2014;16:677-85

18 - Ishak KG, Sesterhenn IA, Goodman ZD, Rabin L, Stromeyer FW. Epithelioid hemangioendothelioma of the liver: a clinicopathologic and follow- up study of 32 cases. Hum Pathol. $1984 ; 15: 839-52$.

19 - Mehrabi A, Kashfi A, Fonouni H, Schemmer P, Schmied BM, Hallscheidt P, et al. Primary malignant hepatic epitheloid hemangioendotelioma: a

comprehensive review of the literature with emphasis on the surgical therapy. Cancer. 2006;107:2108-21.

20 - Makhlouf HR, Ishak KG, Goodman ZD. Epithelioid hemangioendothelioma of the liver: a clinicopathologic study of 137 cases. Cancer. 1999;85:562-82.

21- Frider B, Bruno A, Selser J, Vanesa R, Pascual P, Bistoletti R. Kasabach-Merrit syndrome and adult hepatic epithelioid hemangioendothelioma an unusual association. J Hepatol 2005: 42:282-283.

22- Hayashi Y, Inagaki K, Hirota S, Yoshikawa T, Ikawa H. Epithelioid hemangioendothelioma with marked liver deformity and secondary Budd-Chiari syndrome: pathological and radiological correlation. Pathol Int 1999:49:547-552.

23 - Earnest F 4th1, Johnson CD. Case 96: Hepatic epithelioid hemangioendothelioma. Radiology. $2006 \mathrm{Jul} ; 240(1): 295-8$.

24 - Miller W1, Dodd GD 3rd, Federle MP, Baron RL. Epithelioid hemangioendothelioma of the liver: imaging findings with pathologic correlation. AJR Am J Roentgenol. 1992 Jul;159(1):53-7.

25 - Lyburn ID, Torreggiani WC, Harris AC, Zwirewich CV, Buckley AR, Davis JE, et al. Hepatic epithelioid hemangioendothelioma: sonographic, CT, and MR imaging appearances. AJR Am J Roentgenol. 2003 May;180(5):1359-64.

26 - Paolantonio P1, Laghi A, Vanzulli A, Grazioli L, Morana G, Ragozzino A, et al. MRI of hepatic epithelioid hemangioendothelioma (HEH). J Magn Reson Imaging. 2014 Sep;40(3):552-8.

27- Kim EH, Rha SE, Lee YJ, Yoo IeR, Jung ES, Byun JY.. CT and MR imaging findings of hepatic epithelioid hemangioendotheliomas: emphasis on single nodular type. Abdom Imaging. 2015 Mar;40(3):500-9. 
28 - Bagan $P^{1}$, Hassan M, Le Pimpec Barthes F, Peyrard S, Souilamas R, Danel C, Riquet M. Prognostic factors and surgical indications of pulmonary epithelioid hemangioendothelioma: a review of the literature. Ann Thorac Surg 2006;82:2010-3.

29 - Woo JH, Kim TJ, Lee KS, Kim TS, Kim BT.. Epithelioid hemangioendothelioma in the thorax: Clinicopathologic, CT, PET, and prognostic features. Medicine (Baltimore). 2016 Jul;95(30):e4348.

30 - Kim EY, Kim TS, Han J, Choi JY, Kwon OJ, Kim J.. Thoracic epithelioid hemangioendothelioma: imaging and pathologic features. Acta Radiol. 2011 Mar 1;52(2):161-6.

31- Cronin P, Arenberg D. Pulmonary epithelioid hemangioendothelioma: an unusual case and a review of the literature. Chest 2004;125:789-93.

32- Amin RMS, Hiroshima K, Kokubo T, Nishikawa M, Narita M, Kuroki M, et al. Risk factors and independent predictors of survival in patients with pulmonary epithelioid haemangioendothelioma. Review of the literature and a case report. Respirology. 2006 Nov;11(6):818-25.

33- Sardaro A, Bardoscia L, Petruzzelli MF, Portaluri M. Epithelioid hemangioendothelioma: an overview and update on a rare vascular tumor. Oncol Rev. 23 sept 2014;8(2):259.

34 - Kransdorf M, Murphey MD. Vascular and lymphatic tumors. Imaging of soft-tissue tumors. 2nd edition Philadelphia. Lippincott Williams \& Wilkins 177-88;2006

35 - Angelini A, Mavrogenis AF, Gambarotti M, Angelini A, Mavrogenis AF, Gambarotti M. Surgival treatment and results of 62 patients with epithelioid hemangioendothelioma of bone. J Surg Oncol 2014;109:791-7.

36 - Kleer CG, Unni KK, McLeod RA. Epithelioid hemangioendothelioma of bone. Am J Surg Pathol. nov 1996;20(11):1301-11.

37 - Hettmer S, Andrieux G, Hochrein J, Kurz P, Rössler J, Lassmann S, et al. Epithelioid hemangioendotheliomas of the liver and lung in children and adolescents. Pediatr Blood Cancer. 2017 Dec;64(12).

38 - Deyrup AT, Tighiouart M, Montag AG, Weiss SW. Epithelioid hemangioendothelioma of soft tissue: a proposal for risk stratification based on 49 cases. Am J Surg Pathol 2008;32:924-27

39- Lau K, Massad M, Pollak C, Rubin C, Yeh J, Wang J, et al. Clinical patterns and outcome in epithelioid hemangioendothelioma with or without pulmonary involvement. Chest 2011;140:1312-8.

40 - Gomez-Arellano LI, Ferrari-Caballo T, Dominguez-Malagon HR. Multicentric epithelioid hemangioendothelioma of bone. Report of a case with radiologic-pathologic correlation. Annals Diagn Pathol 2012;16:43-7.

41 - Fagen K, Silverman ED, Cole RL. Detection of a pulmonary epithelioid hemangioendothelioma by FDG PET scan. Clin Nucl Med 2004;29:

$758-9$

42 - Furui S, Itai Y, Ohtomo K, Yamauchi T, Takenaka E, Iio M, et al. Hepatic epithelioid hemangioendothelioma: report of five cases. Radiology 1989;171:63-8.

43 - Watanabe S, Yano F, Kita T, Soga S, Shinmoto H, Kosuda S, et al. 18F-FDG-PET/CT as an indicator for resection of pulmonary epithelioid hemangioendothelioma. Ann Nucl Med 2008;22:521-4.

44- Han JH, Kim TJ, Lee KS, et al. Epithelioid hemangioendothelioma in the thorax: clinicopathologic, CT, PET and prognostic features. Medicine 2016;95:30. 
45 - Dong A, Dong H, Wang Y, Gong J, Lu J, Zuo C. MRI and FDG PET/CT findings of hepatic epithelioid hemangioendothelioma. Clin Nucl Med. 2013;38(2):e66-73.

46 - Lerut JP, Orlando G, Adam R, Schiavo M, Klempnauer J, Mirza D, et al. The place of liver transplantation in the treatment of hepatic epitheloid hemangioendothelioma: report of the European liver transplant registry. Ann Surg. 2007 Dec;246(6):949-57

47 - Grotz TE, Nagorney D, Donohue J, Que F, Kendrick M, Farnell M, et al. Hepatic epithelioid haemangioendothelioma: is transplantation the only treatment option? HPB (Oxford). 2010 Oct;12(8):546-53

48 - Wang Wang LR ${ }^{1}$, Zhou JM, Zhao YM, He HW, Chai ZT, Wang M, et al. Clinical experience with primary hepatic epithelioid hemangioendothelioma: retrospective study of 33 patients. World J Surg. 2012 Nov;36(11):2677-83

49 - Rodriguez JA, Becker NS, O'Mahony CA, Goss JA, Aloia TA. Long-term outcomes following liver transplantation for hepatic hemangioendothelioma: the UNOS experience from 1987 to 2005 . J Gastrointest Surg. 2008 Jan;12(1):110-6.

50 - Kleck CJ, Seidel MJ. Epithelioid hemangioendothelioma of the distal humerus with pathologic fracture. Orthopedics 2012;35:e116-9.

51 - Suga K, Kawakami Y, Hiyama A, Hori K. F-18 FDG PET/CT monitoring of radiation therapeutic effect in hepatic epithelioid hemangioendothelioma. Clin Nucl Med. 2009 Mar;34(3):199-202.

52 - Rojas-Vigott R, Castro CM, Mendez SR. Hemangioendothelioma: A Case Report of Spontaneous Regression. J Thorac Oncol 2016;11(10S):S231-32

53 - Nair LK, Das A, Arun Kumar AS, Ramachandran V. Self Regressing Epitheloid Haemangioendothelioma of Tibia in an Infant- A rare case report and review of literature. Journal of Orthopaedic Case Reports 2015 Jan-March;5(1): 37-40

54 - Otrock ZK, Al-Kutoubi A, Kattar MM, Zaatari G, Soweid A. Spontaneous complete regression of hepatic epithelioid haemangioendothelioma. Lancet Oncol. 2006 May;7(5):439-41.

55- Yousaf N, Maruzzo M, Judson I, Al-Muderis O, Fisher C, Benson C.. Systemic treatment options for epithelioid hemangioendothelioma: the Royal Marsden experience. AntiCancer Research 2015;35:47380

56 - Kelly $\mathrm{H}, \mathrm{O}$ 'Neil BH: Response of epithelioid haemangioendothelioma to liposomal doxorubicin. Lancet Oncol 6:813-815, 2005

57 - Grenader T, Vernea F, Reinus C, Gabizon A. Malignant Epithelioid Hemangioendothelioma of the Liver Successfully Treated With Pegylated Liposomal Doxorubicin. J Clin Oncol VOLUME 29 NUMBER 25 _ SEPTEMBER 12011 e722-24

58 - Pintoffl J; Meisinger I, Mayer F, Horger M, von Weyhern C, Kanz L et al. Long-term disease stabilization during seocn-line gemcitabine in a refractory metastatic haemangioendothelioma. AntiCancer Drugs 2009;20:73-4

59 - Kanemura S, Kuribayashi K, Moriya Y, Shimizu S, Tsujimura T, Nakano T. Pemetrexed for epithelioid haemangioendothelioma of the pleura. Respirol Case Rep. 2016 Nov;4(6):e00191. 
60 - Stacher E, Gruber-Mösenbacher U, Halbwedl I, Dei Tos AP, Cavazza A, Papotti M, et al. The VEGF-system in primary pulmonary angiosarcomas and haemangioendotheliomas: new potential therapeutic targets? Lung Cancer. juill 2009;65(1):49-55.

61 - Raphael C, Hudson E, Williams L, Lester JF, Savage PM. Successful treatment of metastatic hepatic epithelioid hemangioendothelioma with thalidomide: a case report. J Med Case Rep. 2010;4:413.

62 - Kassam A, Mandel K. Metastatic hepatic epithelioid hemangioendothelioma in a teenage girl. J Pediatr Hematol Oncol. 2008;30:550-2.

63 - Kollár A, Jones RL, Stacchiotti S, Gelderblom H, Guida M, Grignani G, et al. Pazopanib in advanced vascular sarcomas: an EORTC Soft Tissue and Bone Sarcoma Group (STBSG) retrospective analysis. Acta Oncol. 2017 Jan;56(1):88-92.

64 - Mascarenhas RC, Sanghvi AN, Friedlander L, Geyer SJ, Beasley HS, Van Thiel DH. Thalidomide inhibits the growth and progression of hepatic epithelioid hemangioendothelioma. Oncology. 2004;67:471-5

65 - Bally O, Tassy L, Richioud B, Decouvelaere A-V, Blay J-Y, Derbel O. Eight years tumor control with pazopanib for a metastatic resistant epithelioid hemangioendothelioma. Clin Sarcoma Res. 2015;5:12.

66 - Chevreau C, Le Cesne A, Ray-Coquard I, Italiano A, Cioffi A, Isambert N, et al. Sorafenib in patients with progressive epithelioid hemangioendothelioma. Cancer 2013;119:2639-44

67 - Sangro B, Iñarrairaegui M, Fernández-Ros N. Malignant epithelioid hemangioendothelioma of the liver successfully treated with Sorafenib. Rare Tumors. 2012 Apr 12;4(2):e34.

68 - Stacchiotti S, Provenzano S, Dagrada G, Negri T, Brich S, Basso U, et al. Sirolimus in Advanced Epithelioid Hemangioendothelioma: A Retrospective Case-Series Analysis from the Italian Rare Cancer Network Database. Ann Surg Oncol. 2016 Sep;23(9):2735-44.

69 - Agulnik M, yarber JL, Okuno SH, von Mehren M, Jovanovic BD, Brockstein BE, et al. An openlabel, multicenter, phase II study of bevacizumab for the treatment of angiosarcoma and epithelioid hemangioendotheliomas. Ann Oncol 2013;24;257-63.

70 - Cioffi A, Italiano, Penel N, Berge Y, Toulmonde M, Salas, et al. Metastatic epitheloid hemangioendothelioma (EHE): Role of systemic therapy and survival. J Clin Oncol 2011;29: Suppl (May 20 2011): 10079-10079

71 - Davies KD, Doebele RC. Molecular pathways: ROS1 fusion proteins in cancer. Clin Cancer Res. 2013;19(15):4040-5. 
Figure 1. Diversité des présentations hépatiques des hémangioendothéliomes épithéloïdes (HEE)

1A. Scanner abdominal en coupe coronale après injection intra-veineuse de produit de contraste iodé, temps portal. Lésion hépatique sous-capsulaire calcifiée, rétractile du dôme hépatique. Contexte: patient de 52 ans. HEE diffus avec atteinte hépatique, lésion évolutive. Chimiothérapie par doxorubicine, stabilité maintenue pendant 13 ans, calcifications secondaires des lésions hépatiques.

1B. Scanner abdominal en coupe axiale après injection intra-veineuse de produit de contraste iodé, temps portal. Infiltration multinodulaire hépatique, avec rétraction capsulaire, lésion pulmonaire postéro-basale droite, splénique et abondante ascite.

Contexte : patient de 60 ans présentant une altération fébrile de l'état général avec ascite. HEE diffus. Décès rapide après échec de lignes de traitement (paclitaxel hebdomadaire et doxorubicine)

1C. Scanner abdominal en coupe axiale sans injection (CT-), puis après injection intra-veineuse de produit de contraste iodé au temps portal $(C T+70 \mathrm{~s})$ et au temps tardif $(C T+5 \mathrm{mn})$. Lésions bifocales du dôme des segments VII-VIII (*) et de segment II ( () . Avant injection, les lésions sont hypodenses. Elles se rehaussent progressivement au temps portal, en motte (flèche blanche), voire s'homogénéise complètement au temps tardif (tête de flèche blanche), comme un angiome hépatique classique.

Contexte: patiente de 60 ans avec initialement atteinte hépatique et vertébrale. Première ligne de traitement par Sorafenib puis $2^{\text {ième }}$ ligne par Doxorubicine devant une progression hépatique et pulmonaire puis $3^{\text {ième }}$ ligne par Endoxan métronomique devant une progression splénique et hépatique et une altération de l'état général. La patient décède de sa maladie 2 ans après le diagnostic.
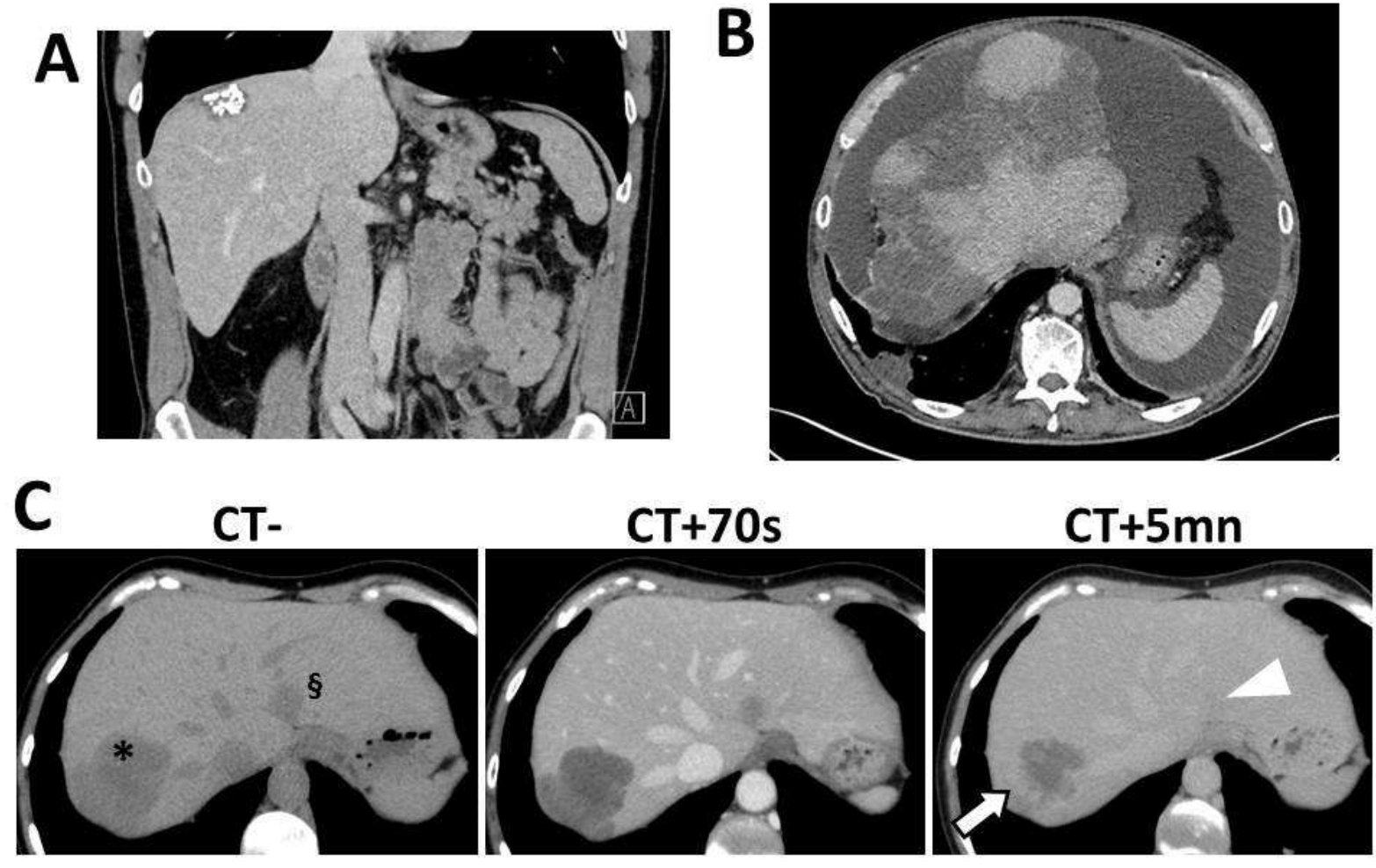
Figure 2. Diversité des présentations thoraciques des hémangioendothéliomes épithéloïdes

2A. Nodule isolé (flèche blanche) de découverte fortuite chez une patiente asymptomatique de 31 ans.

2B. Forme multinodulaire découverte dans un contexte d'asthénie et d'amaigrissement chez un patient de 36 ans. Les nodules sont bilatéraux, asymétriques, juxta-centimétiques, bien limités, aux contours parfois lobulés.

2C. Forme réticulo-nodulaire multifocale symptomatique (toux, hémoptysie) chez un patient de 44 ans avec métastases osseuses et hépatiques. L'atteinte interstitielle affecte aussi bien le poumon central que cortical, sur toute la hauteur du thorax.

2D. Atteinte pleurale gauche (tête de flèche blanche) chez un patient de 28 ans symptomatique (douleur thoracique, dyspnée, altération de l'état général). Noter la rétraction de l'hémithorax gauche.
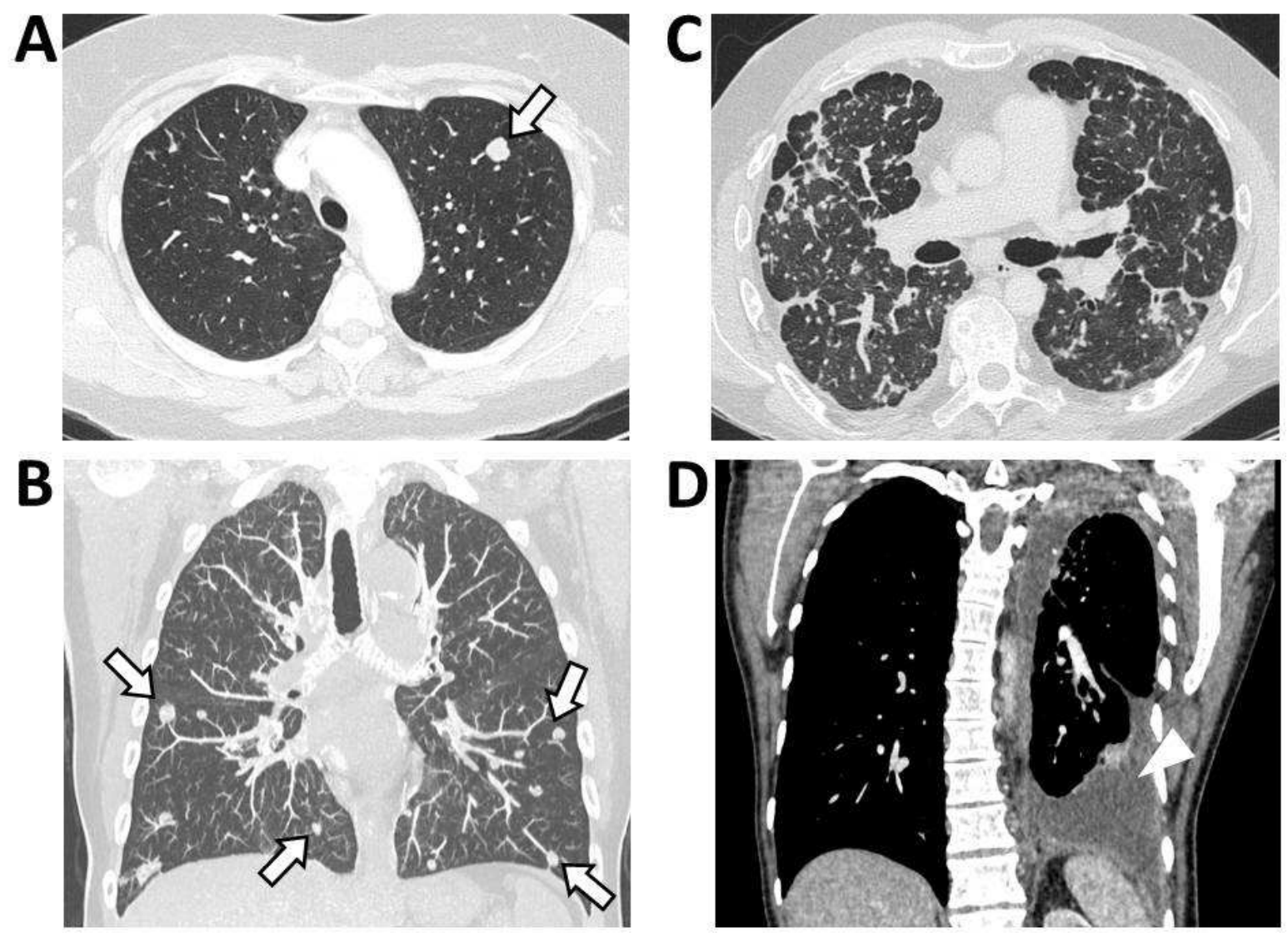
Figure 3. Hémangioendothéliome épithéloïde (HEE) des tissus mous chez une patiente de 46 ans avec métastase hépatiques.

L'examen de référence est alors I'IRM avec au minimum, des séquences pondérées T2 (en coupes coronale (3A) et axiale (3B)), $\mathrm{T} 1(3 \mathrm{C})$ et $\mathrm{T} 1$ avec suppression du signal de la graisse après injection intra-veineuse de chélates de gadolinium (3D). L'hémangioendothéliome épithélioïdeE est de situation profonde, intermusculaire, accolé aux troncs supra-aortiques à droite (flèche blanche). La lésion est de signal hétérogène en $\mathrm{T} 1, \mathrm{~T} 2$ et après injection. Cette atteinte a été responsable d'une thrombose non occlusive de l'axe jugulaire homolatérale. Devant le bon état général et la très lente évolution, une surveillance rapprochée a été préconisée. Deux ans plus tard, les lésions restent parfaitement stables.

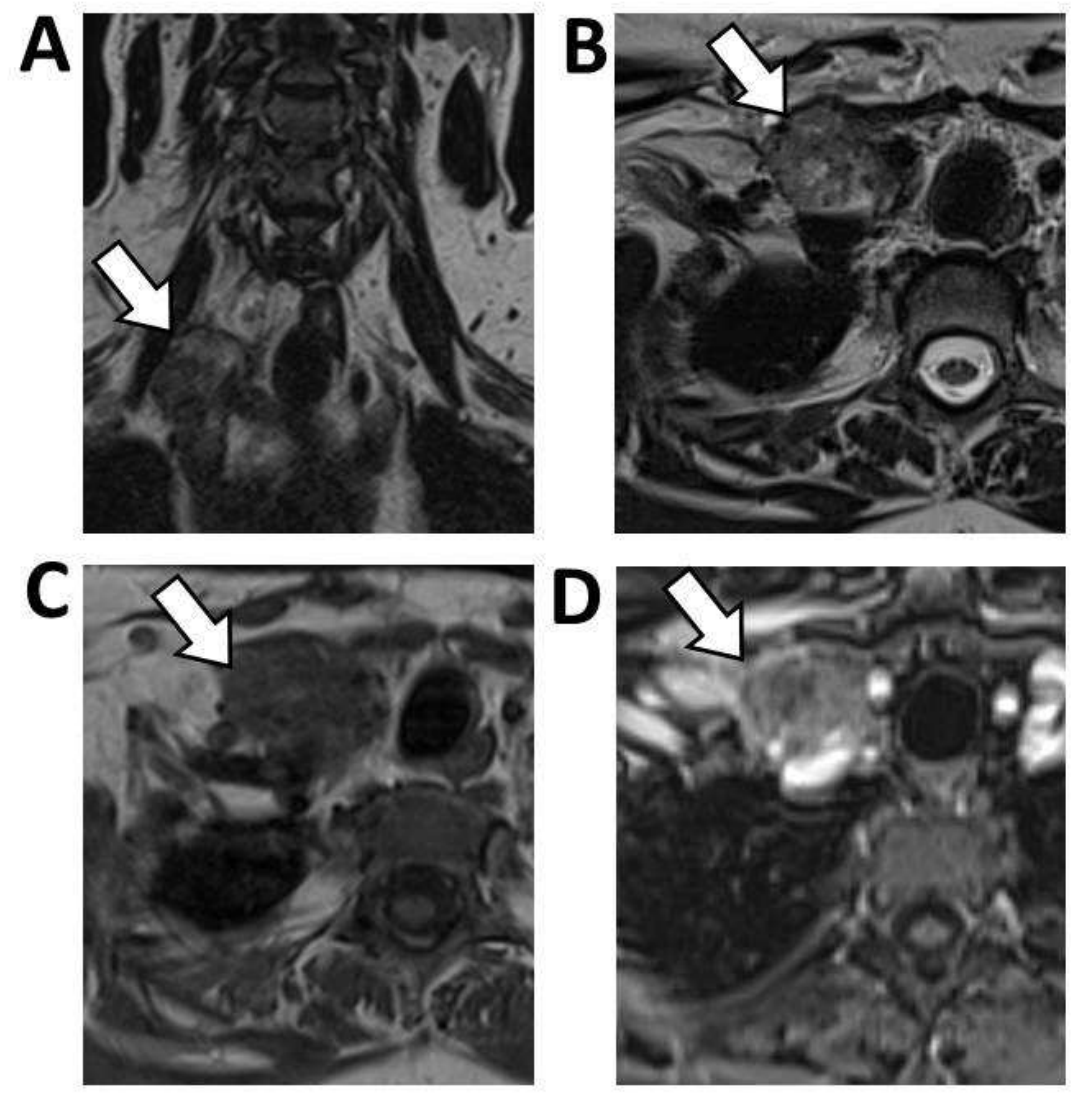


Figure 4. Présentation osseuse symptomatique multifocale d'un hémangioendothéliome épithéloïde chez une patiente de 30 ans.

4A. Radiographies standard de jambe droite de face et de profil montrant des lésions plurifocales ostéolytiques du tibia (tiers supérieur de situation diaphysaire et corticale, tiers inférieur de situation métaphyso-épiphysaire et médullaire) ainsi que de la fibula (tiers supérieur, de situation diaphysaire et médullaire) et du calcaneum (coin postéro-supérieur)

4B. IRM de la cheville droite, séquence sagittale en pondération T1 montrant l'hétérogénéité de signal T1.

4C. Scintigraphie osseuse de face centrée sur les jambes montrant l'hyperfixation des lésions tibiales et fibulaires.

Devant l'importance des douleurs, une irradiation a été réalisée.

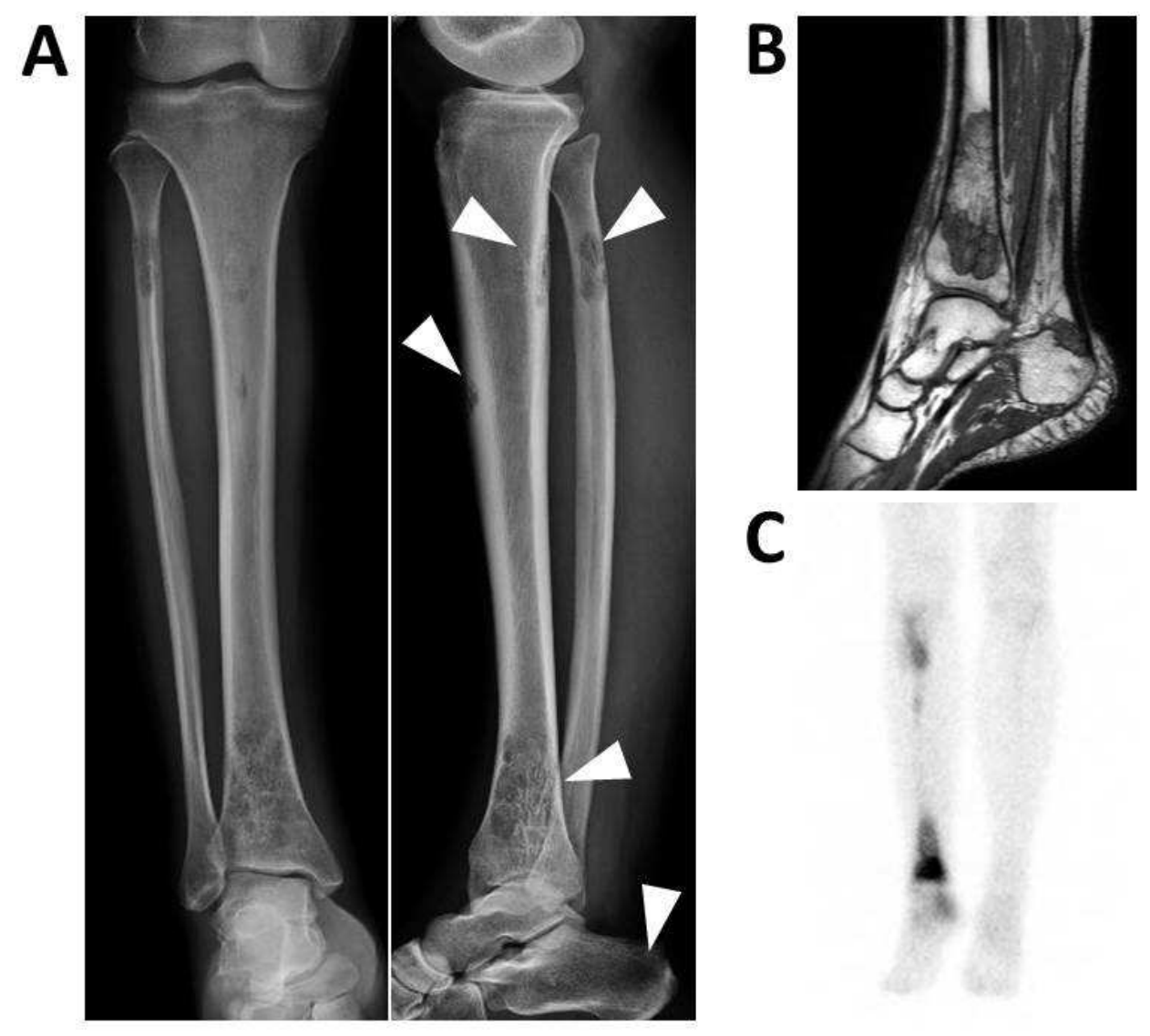


Figure 5. TEP scanner au ${ }^{18}$ FDG et hémangioendothéliomes épithéloïdes (HEE).

La fixation du traceur est inconstante dans ces deux cas, bien que les patients soient tous deux symptomatiques.

5A. Fixation très limitée dans une forme multinodulaire (SUVmax=1.7)

5B. Franche hyperfixation dans une forme mixte réticulonodulaire et multinodulaire (SUVmax $=10.5$ )

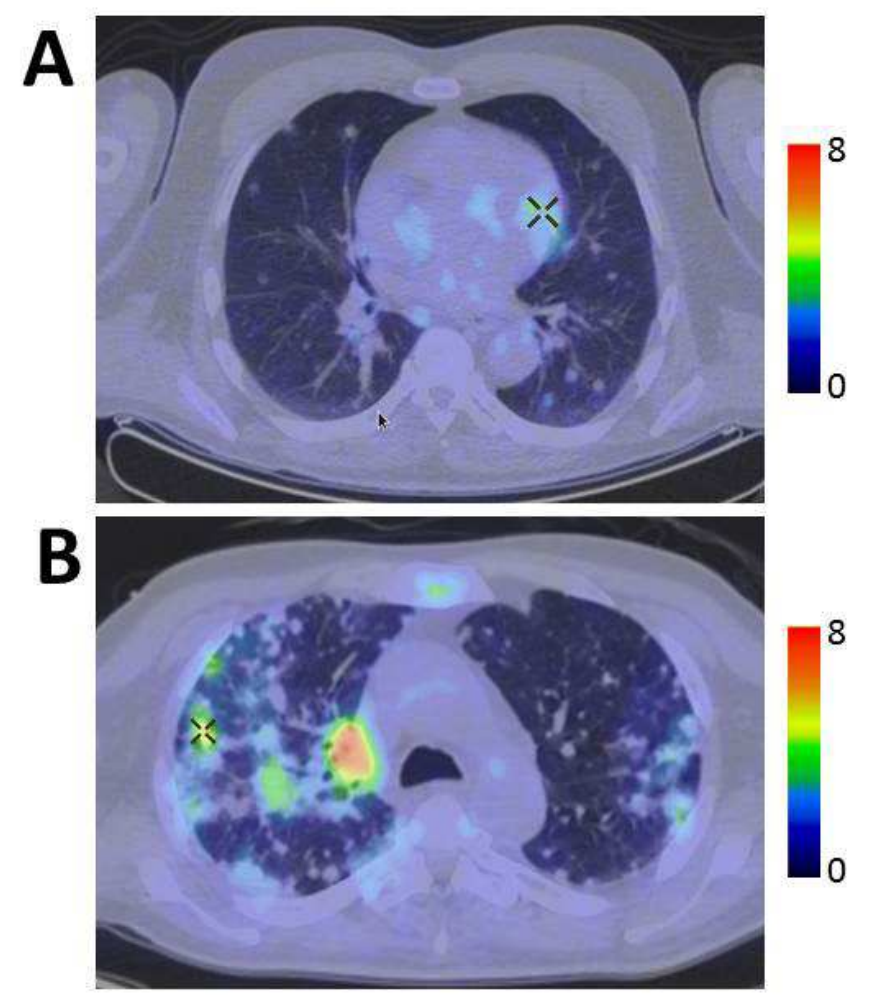


Tableau 1 - Critères diagnostics des hémangioendothéliomes épithélioïdes

\begin{tabular}{|c|c|c|}
\hline & Aspects & Références \\
\hline Macroscopie & $\begin{array}{l}\text { Masse tumorale développée au depend d'une paroi } \\
\text { vasculaire, avec obliteration du vaisseau et extension } \\
\text { centrifuge dans les tissus environnants à partir du } \\
\text { vaisseau }\end{array}$ & {$[2,6]$} \\
\hline Microscopie & $\begin{array}{l}\text { Pas d'aspect de différenciation vasculaire mature mais } \\
\text { phéontype limité vacuoles intracytoplasmiques } \\
\text { contenant des érythrocytes } \\
\text { Cellules tumorales épithélioïdes organisées en chaînes } \\
\text { ou cordes au sein d'un stroma myxo-hyalin } \\
\text { La plupart des HE présentent un aspect nucléaire } \\
\text { monomorphe avec des aspects de bas grade }\end{array}$ & {$[2,6]$} \\
\hline Immuno-histochimie & $\begin{array}{l}\text { Environ } 20 \% \text { des } \mathrm{HE} \text { experiment des marqueurs } \\
\text { vasculaires (ERG, CD31), mais l'expression de CD34 } \\
\text { est inconstante } \\
30 \% \text { des HE expriment des marqueurs épithéliaux } \\
\text { comme Cytokératine } 7 \text { ou } 8 \text {, cytokératine 18, EMA } \\
\text { 90\% des HE expriment CAMTA1 }\end{array}$ & $\begin{array}{l}{[13]} \\
{[14]} \\
{[15]}\end{array}$ \\
\hline Ultra-structure & $\begin{array}{l}\text { Il n'y a pas d'aspect de différenciation vasculaire } \\
\text { mature, mais on peut observer des aspects typiques } \\
\text { de cellules endothéliales avec "basal lumina", vésicules } \\
\text { pinocytiques orientées vers la surface et des corps de } \\
\text { Weibel-Palade. }\end{array}$ & \\
\hline Biologie moléculaire & $\begin{array}{l}\text { 90\% des HE présentent la protéine de fusion WWTR1- } \\
\text { CAMTA1, visibles par méthode FISH ou RT-PCR } \\
10 \% \text { des HE présentent une autre translocation } \\
\text { chromosomique t }(11 ; \mathrm{X})(\mathrm{q} 13 ; \mathrm{p} 11.22) \text { avec uen } \\
\text { protéine de fusion YAP1-TFE3. }\end{array}$ & {$[10]$} \\
\hline
\end{tabular}


Tableau 2 - traitement des hémangioendothéliomes épithélioïdes hépatiques

\begin{tabular}{|l|l|l|l|l|}
\hline Reference & Study & Treatment & $n$ & $\begin{array}{l}\text { Survie à 5 } \\
\text { ans }\end{array}$ \\
\hline Mehrabi [19] & Meta-analyse & Transplantation hépatique & & 55 \\
& & Hépatectomie & 128 & 75 \\
& & Embolisation ou traitement systémique & 60 & 30 \\
& & Observation & 28 & 5 \\
\hline Lerut [46] & Retrospective & Transplantation hépatique & 11 & 80 \\
\hline Grotz [47] & Retrospective & Transplantation hépatique & 11 & 73 \\
& & Hépatectomie & 11 & 86 \\
& & Embolisation ou traitement systémqiue & 8 & 29 \\
\hline Wang [48] & Retrospective & Hépatectomie & 17 & 74 \\
& & Embolisation ou traitement systémique & 13 & 82 \\
\hline Rodriguez [49] & Retrospective & Transplantation hépatique & 100 & 64 \\
\hline Thomas [17] & Retrospective & Hépatectomie & 7 & 83 \\
& & Embolsation ou traitement systémique & 18 & 71 \\
& & Observation & 25 & 72 \\
\hline
\end{tabular}

